\title{
Pediatric Acute Appendicitis Score in Children With Acute Abdominal Pain (AAP)
}

\author{
MAARET ESKELINEN ${ }^{1 *}$, JANNICA MEKLIN $^{1 *}$, KARI SYRJÄNEN $^{2,3}$ and MATTI ESKELINEN ${ }^{1}$ \\ ${ }^{1}$ Department of Surgery, Kuopio University Hospital and School of Medicine, \\ University of Eastern Finland, Kuopio, Finland; \\ ${ }^{2}$ Molecular Oncology Research Center, Barretos Cancer Hospital, Barretos, Brazil; \\ ${ }^{3}$ SMW Consultants, Ltd., Kaarina, Finland
}

\begin{abstract}
Aim: The aim of the study was to compare the accuracy of common clinical findings, laboratory tests and diagnostic score $(D S)$ in diagnosis of clinically confirmed acute appendicitis (AA) in children. Patients and Methods: A cohort of 188 children under the age of 15 years presenting with acute abdominal pain (AAP) were included in the study. The clinical history $(n=21)$, signs $(n=14)$ and tests $(n=3)$ were recorded in each child. Results: The significant independent diagnostic predictors (disclosed by multivariate logistic regression model) were used to construct the DS formulas for AA diagnosis. These formulas were tested at six different cut-off levels to establish the most optimal diagnostic performance for clinically confirmed $A A$. In the receiver operating characteristic curve (ROC) comparison test, there was a statistically significant difference $(p=0.0055)$ in the area under curve (AUC) values between $i)$ clinical history and symptoms (AUC=0.594), and ii) signs and laboratory tests $(A U C=0.734)$, whereas both were significantly inferior $(p=0.0001)$ to the $A U C$ value of the DS (AUC=0.952). Conclusion: In diagnosis of clinically confirmed $A A$ in children, the DS formula is superior to both the clinical history/symptoms and signs/lab tests, justifying the use of DS as an integral part of the diagnostic algorithm of $A A$ in all children presenting with $A A P$.
\end{abstract}

Although, the incidence of acute appendicitis (AA) has been quite stable in Western countries, the incidence of AA is

This article is freely accessible online.

*These Authors contributed equally to this study.

Correspondence to: Matti Eskelinen, MD, Ph.D., School of Medicine, University of Eastern Finland, P.O. Box 100, FI-70029 KYS, Finland. Tel: +358 17173311, Fax: +358 17172611, GSM: +358 400969444, e-mail: matti.eskelinen@kuh.fi

Key Words: Acute appendicitis, acute abdominal pain, diagnostic score, children, ROC, HSROC, diagnostic accuracy. rapidly rising in the newly industrialized countries $(1,2)$. AA is confirmed clinically based on symptoms, signs and diagnostic tests. Ultrasonography (US) examination, leucocyte count (LC) and other inflammatory markers are often performed to confirm the diagnosis of AA. However, AA with appendectomy still remains to be confirmed in an operation performed without full certainty of the definitive AA diagnosis. Although, the clinical symptoms, signs and clinical tests do not establish AA diagnosis with certainty, it is necessary to determine which of the children presenting with acute abdominal pain (AAP) need immediate surgical intervention (appendectomy) and for whom follow-up is a safe conduct.

Several diagnostic scores (DS) have been developed to differentiate AA from other causes of AAP. Some DS systems could increase the diagnostic accuracy in AA and decrease the false-positive appendectomy rate (3-10). Lintula et al. $(6,8)$ have previously constructed and validated a DS for AA in children, but unfortunately, they did not use hierarchical summary receiver operating characteristic (HSROC) and area under curve (AUC) analysis to confirm the diagnostic performance of DS in these children $(6,8)$. Prompted by the difficulty of AA diagnosis among children and the lack of diagnostic performance studies on DS (with HSROC analysis), we designed the present study to assess the relative accuracy of i) a detailed history taking, ii) clinical examination and laboratory testing, and iii) the DS in detecting clinically confirmed AA among the children with AAP.

\section{Patients and Methods}

Criteria for inclusion in the study and diagnostic criteria used were those set by the Research Committee of the World Organization of Gastroenterology (OMGE) (11-14). Included in the present study of 188 children under the age of 15 years were 93 girls $(49.5 \%)$ and 95 boys $(50.5 \%)$ with the mean age $( \pm \mathrm{SD})$ of $9.6 .0 \pm 3.0$ years.

The diagnosis of AA was done by considering all symptoms, signs and results of the laboratory tests weighted against the accepted diagnostic criteria of AA (Tables I and II) (12-14). 
Table I. The clinical history of the patients with acute appendicitis versus other cause of abdominal pain in children.

\begin{tabular}{|c|c|c|c|c|c|c|}
\hline Clinical history variable & Positive endpoint & Negative endpoint & $\mathrm{TP}$ & FN & FP & $\mathrm{TN}$ \\
\hline 1. Location of initial pain & $\begin{array}{l}\text { Upper left or right } \\
\text { quadrants of abdomen }\end{array}$ & $\begin{array}{l}\text { Other quadrants } \\
\text { of abdomen }\end{array}$ & 7 & 37 & 29 & 115 \\
\hline 2. Location of pain at diagnosis & $\begin{array}{l}\text { Right lower quadrant } \\
\text { of abdomen }\end{array}$ & $\begin{array}{l}\text { Other quadrants } \\
\text { of abdomen }\end{array}$ & 32 & 12 & 45 & 99 \\
\hline $\begin{array}{l}\text { 3. Duration of pain: } \\
\text { Duration of pain at diagnosis }\end{array}$ & $\leq 48$ hours & $>48$ hours & 38 & 6 & 107 & 37 \\
\hline 4. Intensity of abdominal pain & $\begin{array}{l}\text { Subjectively moderate/ } \\
\text { intolerable pain }\end{array}$ & Weak pain & 26 & 18 & 60 & 84 \\
\hline $\begin{array}{l}\text { 5. Progression of pain from } \\
\text { onset to diagnosis }\end{array}$ & $\begin{array}{l}\text { Subjectively same } \\
\text { or worse pain }\end{array}$ & $\begin{array}{l}\text { Weaker pain than } \\
\text { at the onset }\end{array}$ & 33 & 11 & 90 & 54 \\
\hline 6. Type of pain & Subjectively steady pain & Colicky or intermittent pain & 28 & 16 & 77 & 67 \\
\hline 7. Aggravating factors & $\begin{array}{l}\text { Movement, coughing, } \\
\text { respiration, food or other }\end{array}$ & No aggravating factors & 39 & 5 & 90 & 54 \\
\hline 8. Relieving factors & $\begin{array}{l}\text { No relieving } \\
\text { factors }\end{array}$ & $\begin{array}{l}\text { Vomiting, lying still, } \\
\text { food, antacids or } \\
\text { no relieving factors }\end{array}$ & 7 & 37 & 52 & 92 \\
\hline 9. Previous similar pain & No & Yes & 38 & 5 & 107 & 35 \\
\hline 10. Vertigo & No & Yes & 44 & 0 & 142 & 2 \\
\hline 11. Nausea & Yes & No & 27 & 17 & 69 & 75 \\
\hline 12. Vomiting & Yes & No & 32 & 12 & 51 & 93 \\
\hline 13. Appetite & No appetite & Normal appetite & 36 & 8 & 103 & 41 \\
\hline 14. Previous indigestion & No & Yes & 42 & 2 & 134 & 10 \\
\hline 15. Jaundice & No & Yes & 44 & 0 & 144 & 0 \\
\hline 16. Bowels & Normal & $\begin{array}{l}\text { Constipation, diarrhea, } \\
\text { lood, mucus, white } \\
\text { or normal stools }\end{array}$ & 37 & 7 & 117 & 27 \\
\hline 17. Micturition & Normal & Abnormal & 42 & 2 & 141 & 3 \\
\hline 18. Drugs for abdominal pain & No & Yes & 44 & 0 & 142 & 1 \\
\hline 19. Previous abdominal surgery & No & Yes & 44 & 0 & 140 & 3 \\
\hline 20. Previous abdominal diseases & No & Yes & 2 & 42 & 5 & 138 \\
\hline 21. Use of alcohol & No & Yes & 44 & 0 & 143 & 0 \\
\hline
\end{tabular}

TP: True positive; FN: false negative; FP: false positive; TN: true negative.

Table II. The clinical signs and investigations of patients with acute appendicitis versus other cause of abdominal pain in children.

\begin{tabular}{|c|c|c|c|c|c|c|}
\hline Clinical signs and investigations & Positive endpoint & Negative endpoint & $\mathrm{TP}$ & $\mathrm{FN}$ & FP & $\mathrm{TN}$ \\
\hline 1. Mood & Distressed or anxious & Normal & 9 & 35 & 24 & 120 \\
\hline 2. Colour & Normal, flushed & Jaundiced, pale or cyanosed & 43 & 1 & 138 & 6 \\
\hline 3. Abdominal movement & Poor/nil & Normal & 5 & 39 & 4 & 140 \\
\hline 4. Scar & No & Yes & 44 & 0 & 141 & 3 \\
\hline 5. Distension & No & Yes & 44 & 0 & 143 & 1 \\
\hline 6. Tenderness & $\begin{array}{c}\text { Right lower quadrant } \\
\text { of abdomen }\end{array}$ & $\begin{array}{l}\text { Other quadrants } \\
\text { of abdomen }\end{array}$ & 38 & 6 & 55 & 89 \\
\hline 7. Mass & No & Yes & 44 & 0 & 143 & 1 \\
\hline 8. Rebound & Yes & No & 40 & 4 & 50 & 94 \\
\hline 9. Guarding & Yes & No & 38 & 6 & 43 & 101 \\
\hline 10. Rigidity & Yes & No & 28 & 16 & 8 & 135 \\
\hline 11. Murphy's positive & Yes & No & 3 & 41 & 0 & 144 \\
\hline 12. Bowel sounds & Abnormal & Normal & 10 & 34 & 8 & 136 \\
\hline 13. Renal tenderness & No & Yes & 35 & 9 & 122 & 22 \\
\hline 14. Rectal digital tenderness & Abnormal & Normal & 27 & 17 & 47 & 97 \\
\hline 15. Body temperature (Temp) & $\geq 37.1^{\circ} \mathrm{C}$ & $<37.1^{\circ} \mathrm{C}$ & 31 & 13 & 77 & 63 \\
\hline 16. Leucocyte count (LC) & $\geq 10000 / \mathrm{mm}^{3}$ & $<10000 / \mathrm{mm}^{3}$ & 34 & 9 & 38 & 82 \\
\hline 17. Urine & Normal & Haematuria or bacteriuria & 43 & 0 & 125 & 3 \\
\hline
\end{tabular}

TP: True positive; FN: false negative; FP: false positive; TN: true negative. 


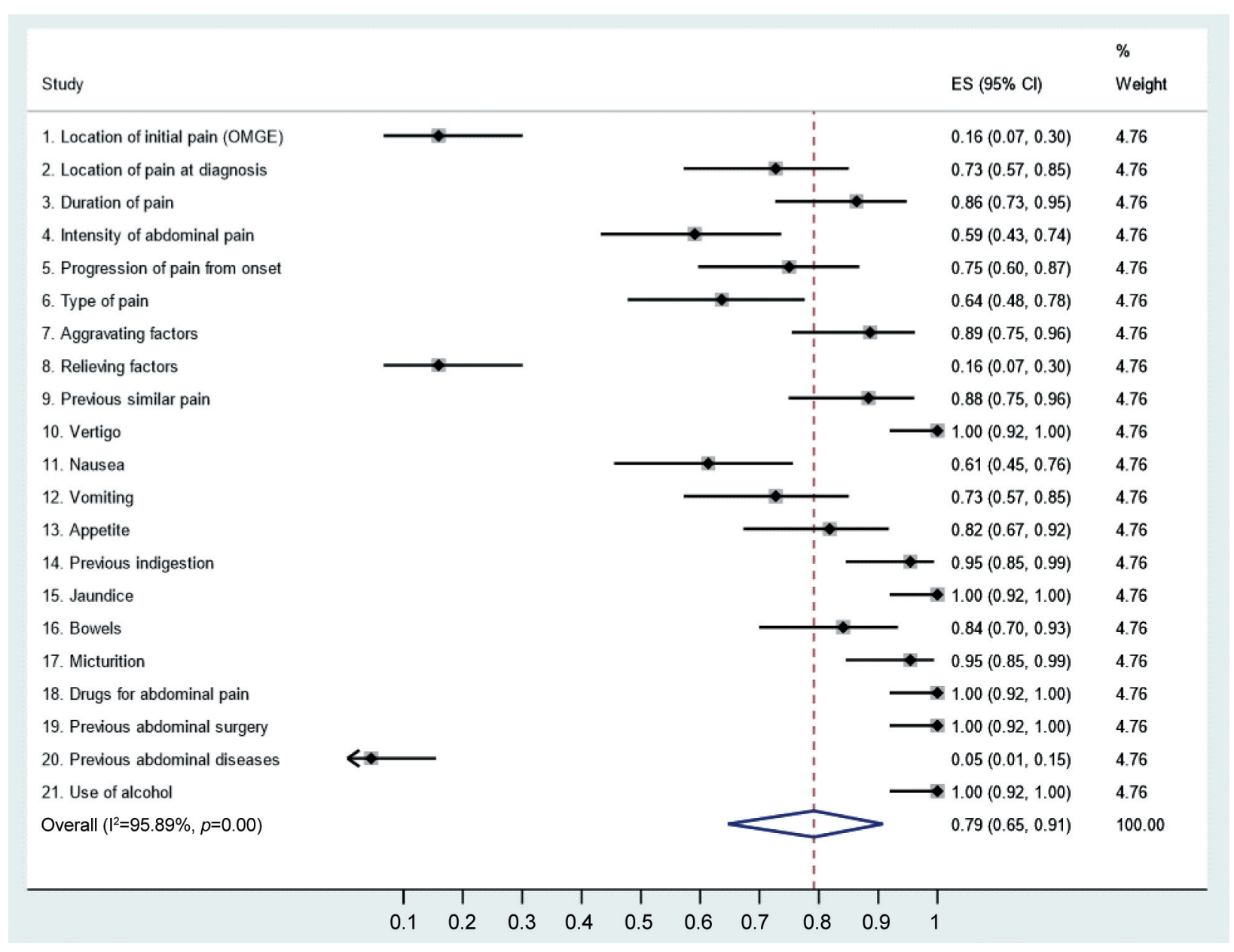

Figure 1. Pooled sensitivities of the clinical symptoms in acute appendicitis in children (random-effects model). ES: Estimated sensitivity; CI: confidence interval.

Table III. Diagnostic score for acute appendicitis in children shown at six different cut-off levels of symptoms, signs and tests. Cut-off levels: DS $I=-1.15$, DS II=-1.13, DS III=-0.29, DS IV=DS values between -1.15 and -0.29 excluded, $n=25$ patients, DS V=DS values between -1.15 and -1.13 excluded, $n=14$ patients, $D S$ VI=DS values between -1.13 and -0.29 excluded, $n=11$ patients.

\begin{tabular}{|c|c|c|c|c|c|c|}
\hline Diagnostic score (DS) & Positive endpoint & Negative endpoint & $\mathrm{TP}$ & FN & FP & $\mathrm{TN}$ \\
\hline 1. Logistic model DS I & Appendicitis & Other cause of abdominal pain & 42 & 2 & 30 & 123 \\
\hline 2. Logistic model DS II & Appendicitis & Other cause of abdominal pain & 39 & 5 & 19 & 124 \\
\hline 3. Logistic model DS III & Appendicitis & Other cause of abdominal pain & 35 & 9 & 12 & 131 \\
\hline 4. Logistic model DS IV & Appendicitis & Other cause of abdominal pain & 35 & 2 & 12 & 113 \\
\hline 5. Logistic model DS V & Appendicitis & Other cause of abdominal pain & 39 & 2 & 17 & 115 \\
\hline 6. Logistic model DS VI & Appendicitis & Other cause of abdominal pain & 35 & 5 & 12 & 124 \\
\hline
\end{tabular}

*Logistic regression analysis formula for DS: $1.752 \times$ location of pain at diagnosis (positive endpoint $=1$, negative endpoint $=0$ ) $+1.728 \times$ vomiting (positive endpoint $=1$, negative endpoint $=0)+2.192 \times$ guarding (positive endpoint $=1$, negative endpoint $=0)+3.061 \times$ rigidity $($ positive endpoint $=1$, negative endpoint $=0)-5.074$. TP: True positive; FN: false negative; FP: false positive; TN: true negative.

Identifying the DS models. As the first step in constructing the DS, a multivariate logistic (stepwise) regression analysis (SPSS Statistics 26.0.0.1; IBM, NY, USA) was performed to disclose the variables with an independent predictive value. All the variables presented in Tables I and II were included in the analysis as binary data e.g. $\mathrm{AA}=1$ and other diagnosis of $\mathrm{AAP}=0$. Using the coefficients of the regression model, a DS was built and its predictive value for AA was studied (Table III). The coefficient of the multivariate analysis shows the relative risk $\left(R R=e_{-}, n=\beta\right)$ of a patient with a given symptom or sign to have an AA. 


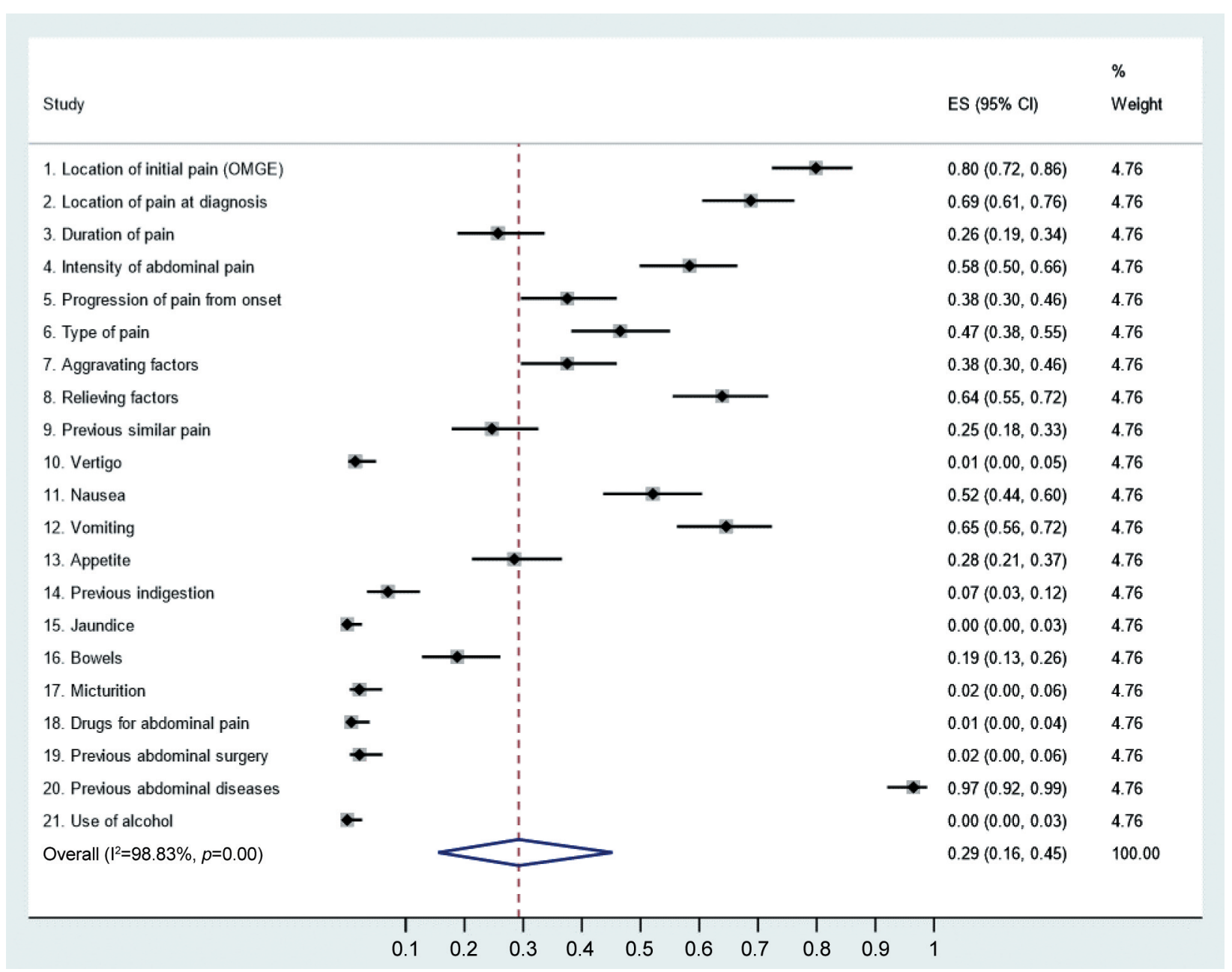

Figure 2. Pooled specificities of the clinical symptoms in acute appendicitis in children (random-effects model). ES: Estimated specificity; CI: confidence interval.

The DS formula for AA in children. The DS formula for AA in children, showing the highest diagnostic performance for AA in HSROC analysis is as follows DS: $1.752 \times$ location of pain at diagnosis (positive endpoint $=1$, negative endpoint $=0$ ) $+1.728 \times$ vomiting (positive endpoint $=1$, negative endpoint $=0$ ) $+2.192 \times$ guarding (positive endpoint $=1$, negative endpoint $=0$ ) $+3.061 \times$ rigidity (positive endpoint $=1$, negative endpoint $=0$ ) -5.074 . The DS values ranged between -5.07 and 3.66 and the mean (SD) of the DS values for children with AA $(n=188)$ were $-2.06( \pm 2.6)$.

Statistical analysis. All other statistical analyses were performed using STATA/SE version 16.1 (StataCorp, College Station, TX, USA). Statistical tests presented were two-sided, and p-value $<0.05$ was considered statistically significant. Using $2 \times 2$ tables, we calculated sensitivity (Se) and specificity ( $\mathrm{Sp}$ ) with $95 \%$ confidence intervals $(95 \% \mathrm{CI})$ for each symptom, sign or laboratory test, and created separate forest plots for showing each set of data, separately for each diagnostic variables. We calculated the summary estimates of $\mathrm{Se}$ and $\mathrm{Sp}$, positive likelihood ratio $(\mathrm{LR}+)$ and negative likelihood ratio (LR-) and diagnostic odds ratio (DOR), using a random effect bivariate model and fitted the summary hierarchical receiver operating characteristic (HSROC) curves, including all diagnostic variables in the DS model, using the AA endpoint.

Using the STATA's prediction tool, we also made posterior predictions (EB=Empirical Bayes estimates) of the Se and $\mathrm{Sp}$ for each diagnostic variable in AA patients, including the different DS cut-offs. Analogous to its use in meta-analysis, EB estimates here give the best estimates of the true Se and $\mathrm{Sp}$ for each diagnostic variable, the variable-specific point estimates usually shrinking toward the summary point of the HSROC. We explored the statistical heterogeneity between diagnostic variables and DS models through visual examination of the forest plots and the HSROC curves.

\section{Results}

Diagnostic performance of the symptoms. The pooled overall $\mathrm{Se}$ of the diagnostic symptoms in confirming AA in children was $79 \%(95 \% \mathrm{CI}=65 \%-91 \%$ ) (Figure 1$)$. Se exceeded $79 \%$ for 12 diagnostic symptoms, and the five best diagnostic 


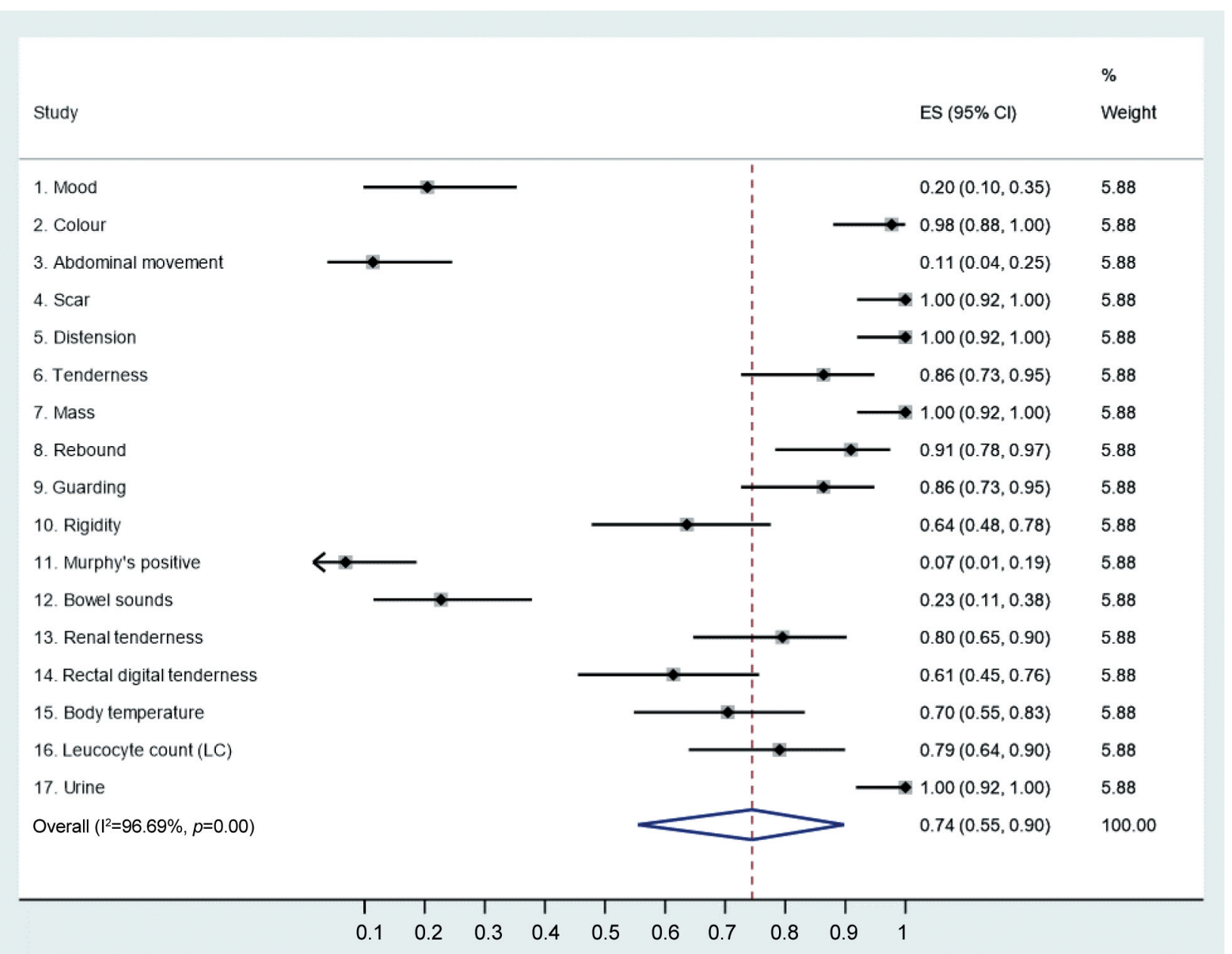

Figure 3. Pooled sensitivities of the clinical signs and tests in acute appendicitis in children (random-effects model). ES: Estimated sensitivity; CI: confidence interval.

symptoms (vertigo, jaundice, drugs for abdominal pain, previous abdominal surgery, type of pain) showed $100 \% \mathrm{Se}$ in diagnosis of AA (Figure 1). The pooled overall $\mathrm{Sp}$ of the symptoms for detecting AA was only $29 \%$ (95\% CI=16\%$45 \%$ ) (Figure 2). Ten symptoms showed Sp higher than $29 \%$, whereas the best five symptoms (location of initial pain, location of pain at diagnosis, relieving factors, vomiting, previous abdominal diseases) showed $\mathrm{Sp}$ varying between 64-97\% Sp (Figure 2).

Diagnostic performance of the signs and tests. The pooled overall Se of the signs and tests for detecting AA in children was $74 \%(95 \% \quad \mathrm{CI}=55 \%-90 \%)$ (Figure 3), while ten diagnostic signs and tests had Se exceeding $74 \%$. The five most accurate signs and tests (colour, scar, distension, mass and urine) showed Se in the range of $98-100 \%$ Se (Figure 3). The pooled overall $\mathrm{Sp}$ of the signs and tests was only $50 \%$ (95\% CI $=27 \%-73 \%$ ) (Figure 4), and ten signs and tests showed Sp higher than $50 \%$. The five most accurate diagnostic signs and tests (mood, abdominal movement, rigidity, Murphy's positive and bowel sounds) had Sp of 83$100 \%$ (Figure 4).

Diagnostic performance of the DS formulas. The pooled overall Se of the DS formulas for AA in children was $91 \%$ (95\% CI=85-95\%). The best three DS models (DS I, DS IV and DS V) had Se of 95\%. The pooled overall Sp of the DS formulas for confirming AA was $88 \%$ (95\% CI=85-91\%), with the best three DS models (DS III, DS IV and DS VI) with Sp of 90-92\% (Figures 5 and 6).

HSROC analyses and empirical Bayes (EB) estimates. STATA (metandiplot algorithm) was used to draw the HSROC curves and EB estimates to visualise and compare the pooled overall diagnostic performance of the different symptoms, signs, tests and the DS formulas in diagnosis of 


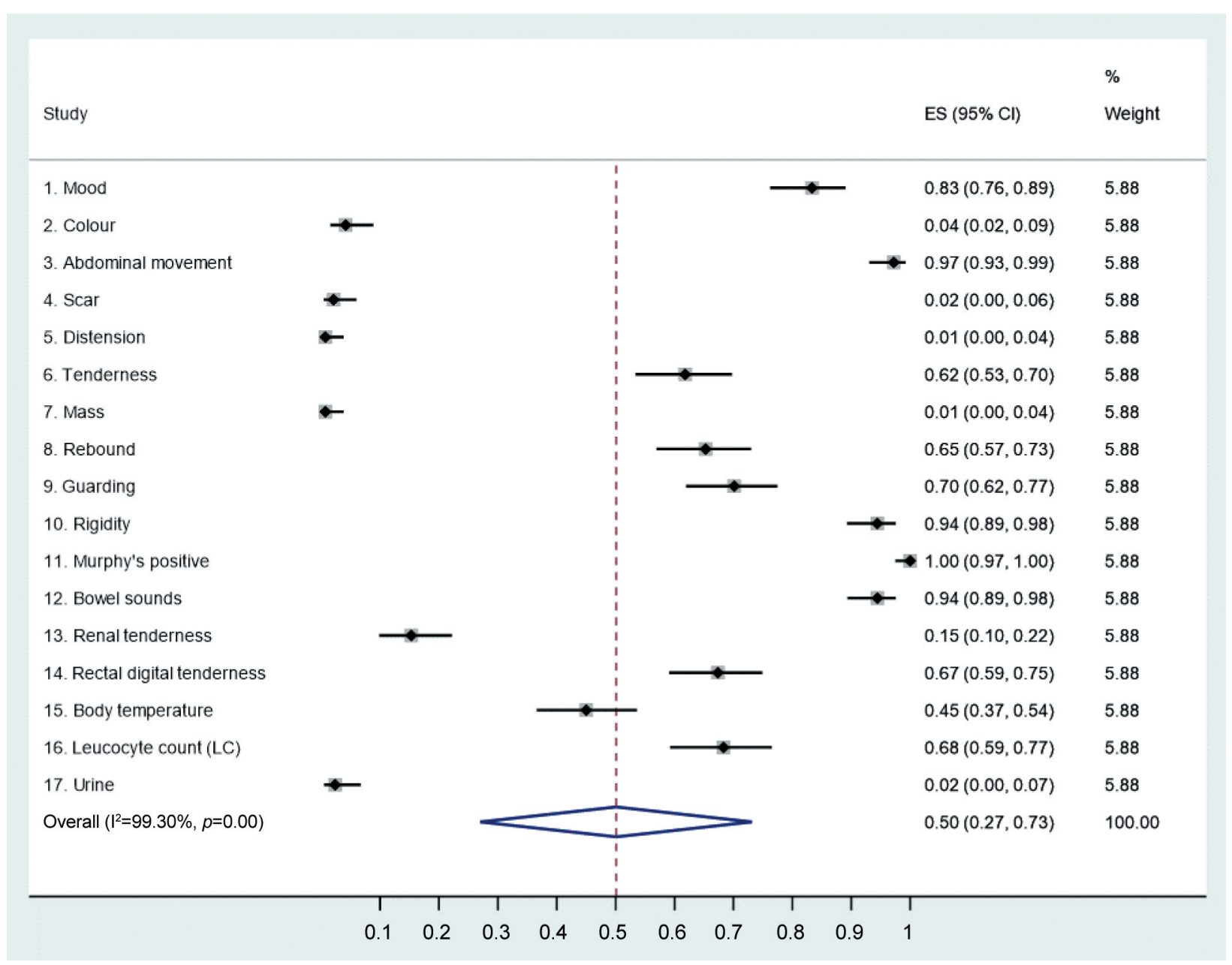

Figure 4. Pooled specificities of the clinical signs and tests in acute appendicitis in children (random-effects model). ES: Estimated specificity; CI: confidence interval.

AA (Figures 5, 6, 7, 8 and 9). Based on comparisons of the HSROC AUC values, i) the common clinical findings, as well as ii) signs and tests, were significantly inferior to iii) the AUC values reached by the DS formulas as follows: between Figure 7 (AUC $=0.594,95 \% \mathrm{CI}=0.550-0.634)$ and Figure 8 (AUC $=0.734,95 \% \quad \mathrm{CI}=0.680-0.788$ ), $p=0.0055$ (ROC comparison test); between Figure 7 (see above) and Figure 9 (AUC $=0.952,95 \% \mathrm{CI}=0.930-0.968), p=0.0001$; between Figure 8 and Figure 9, the difference is also highly significant $(p=0.0001)$.

\section{Discussion}

The diagnosis of AA in children is traditionally made on the basis of common clinical findings, supported by signs and laboratory tests $(15,16)$. Clinical findings of AA in children include right lower quadrant pain and tenderness, nausea, vomiting, fever and poor appetite. The differential diagnosis of AA in children can be difficult and may include several different diseases (11-14).

Albeit AA is a common cause of AAP in children, the accuracy of DS in diagnosis of AA in children has not been critically evaluated $(15,16)$. To cast further light on this issue, the present study was designed to conduct a detailed analysis of the relative accuracy of i) the common clinical findings, ii) signs and tests, as compared with iii) the DS, to establish whether the DS could improve the diagnostic accuracy of AA in children. Of interest was to compare the diagnostic performance of the symptoms, signs and tests among the AA in children to those of adult AA patients, reported in our recent study (14), to see whether the diagnostic accuracy of common clinical findings differs in AA in children and adults. Indeed, this seems to be the case in that the pooled Se of the symptoms in AA in children $79 \%$ (95\% CI=65-91\%) was 


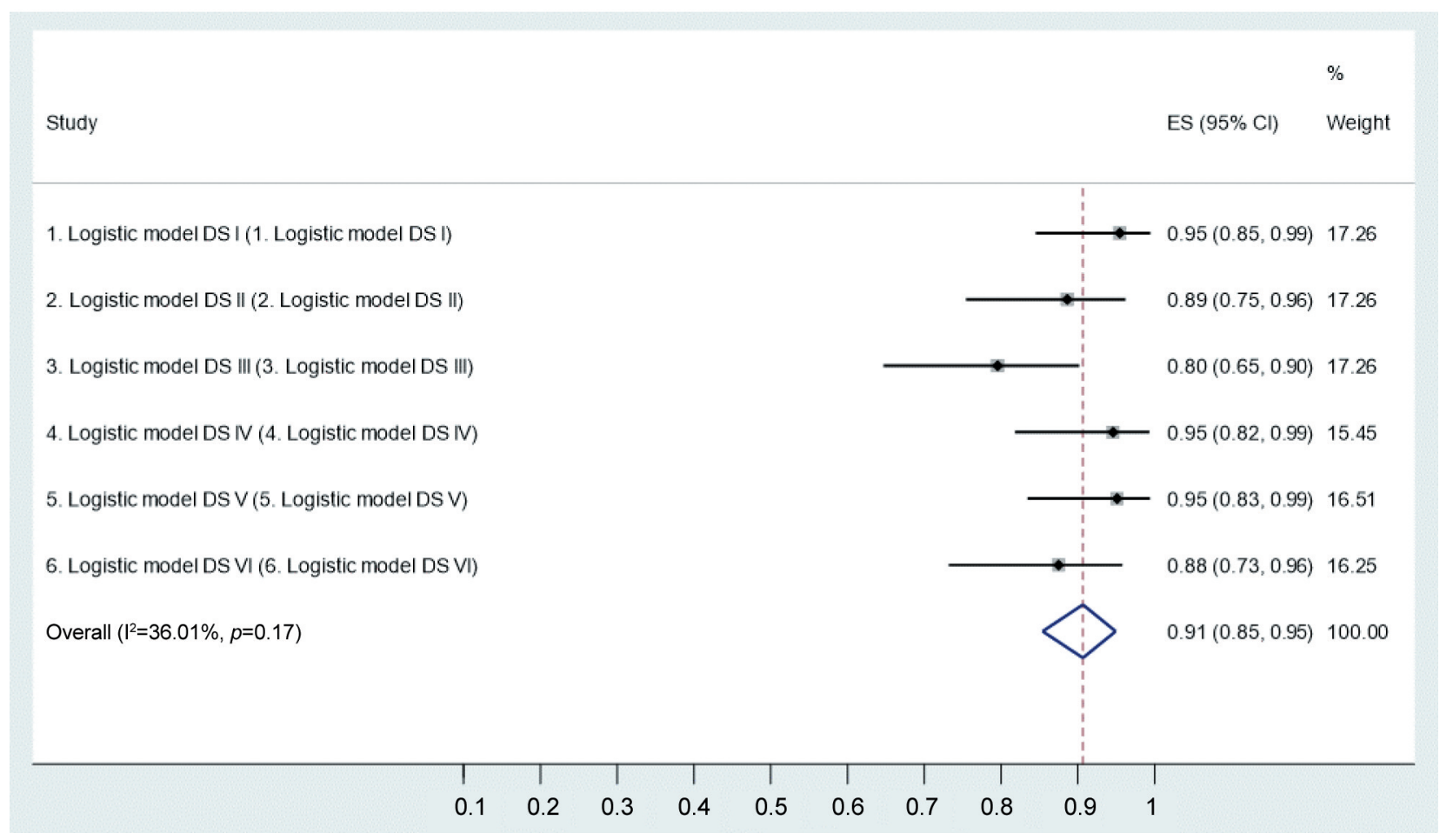

Figure 5. Sensitivities of diagnostic scores at six different cut-off levels (DS I-VI).

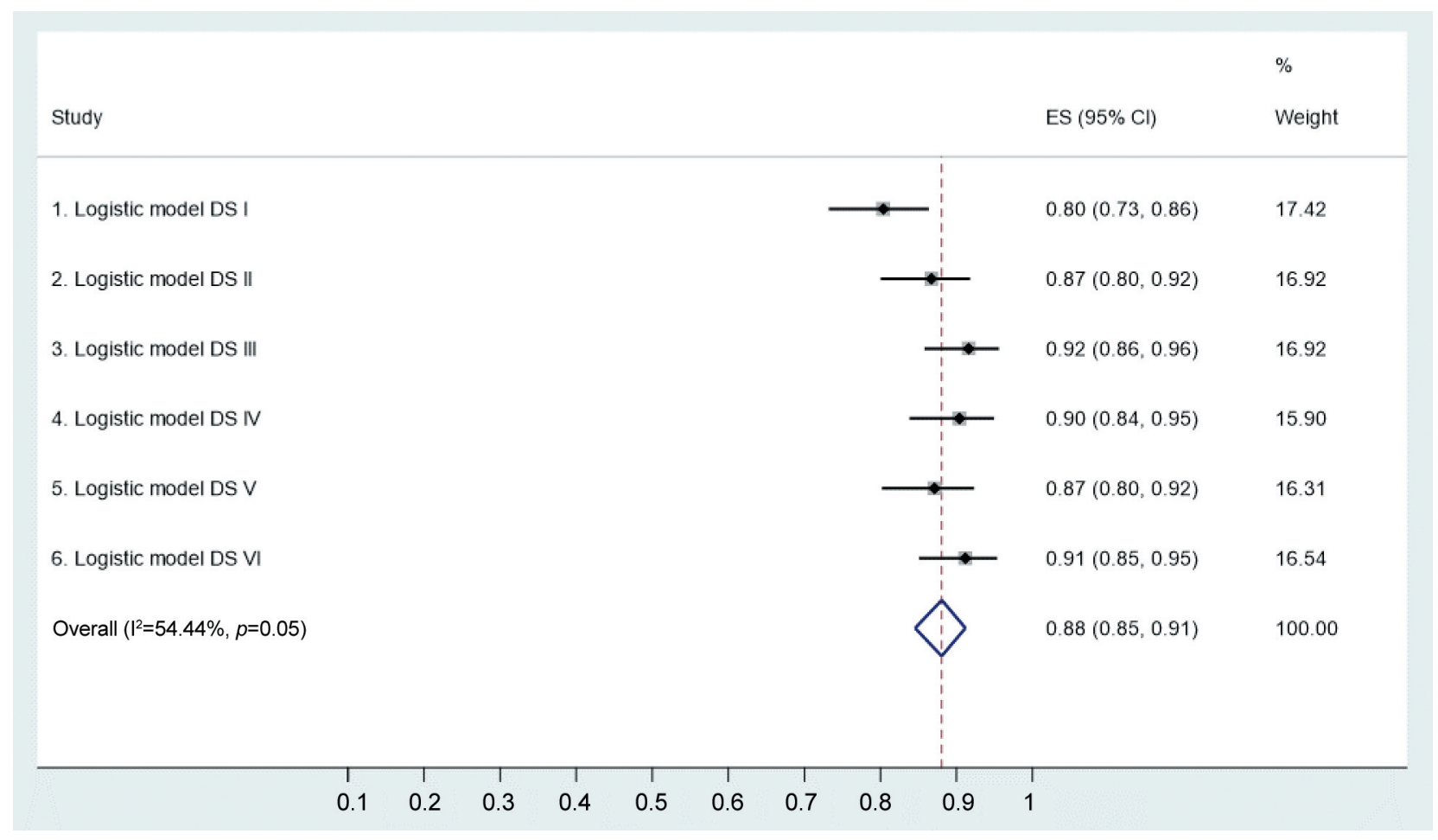

Figure 6. Specificities of diagnostic scores at six different cut-off levels (DS I-VI). 


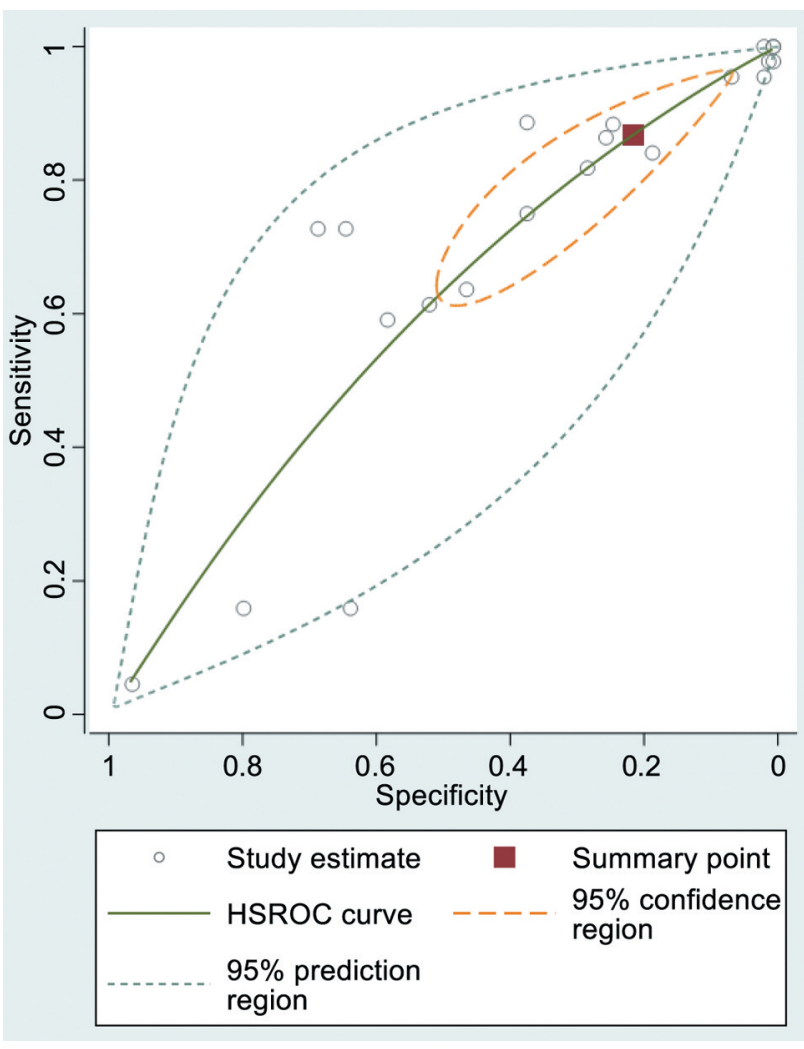

Figure 7. Hierarchical summary receiver operating characteristic (HSROC) curve of the symptoms.

slightly lower that in AA in adult female and male AA patients; $80 \%(95 \% \mathrm{CI}=67-90 \%)$ and $81 \%(95 \% \mathrm{CI}=66-92 \%)$ respectively. However, the pooled overall $\mathrm{Sp}$ of the symptoms in diagnosis of AA in children was $29 \%$ (95\% CI=16-45\%) which was slightly lower than in detecting AA in adult female and male patients; $30 \%(95 \% \mathrm{CI}=19-42 \%)$ and $31 \%(95 \%$ $\mathrm{CI}=20-43 \%$ ), respectively. Similarly, the pooled overall Se of the signs and tests in diagnosis of AA in children was significantly lower, $74 \%$ (95\% CI=55-90\%), than that for adult female and male patients; $86 \%(95 \% \mathrm{CI}=79-92 \%)$ and $88 \%$ (95\% CI=82-94\%), respectively. As anticipated, however, the pooled overall $\mathrm{Sp}$ of the signs and tests for AA in children was higher, $50 \%$ (95\% CI=27-73\%) than that in detecting AA in adult female and male patients; $34 \%(95 \% \mathrm{CI}=20-50 \%)$ and $34 \%$ (95\% CI=20-51\%), respectively.

When the same comparisons were calculated for the diagnostic accuracy of the DS formulas between children and adult AA patients from our earlier study (14), the trend is quite similar. Indeed, the pooled Se of the DS formulas is quite similar in children $91 \%(95 \% \mathrm{CI}=85-95 \%)$ than in adult female and male patients; $90 \%(95 \% \mathrm{CI}=85-95 \%)$ and $93 \%$ (95\% CI=88-96\%), respectively. Because Se and Sp behave reciprocally, it was not unexpected to find that the pooled

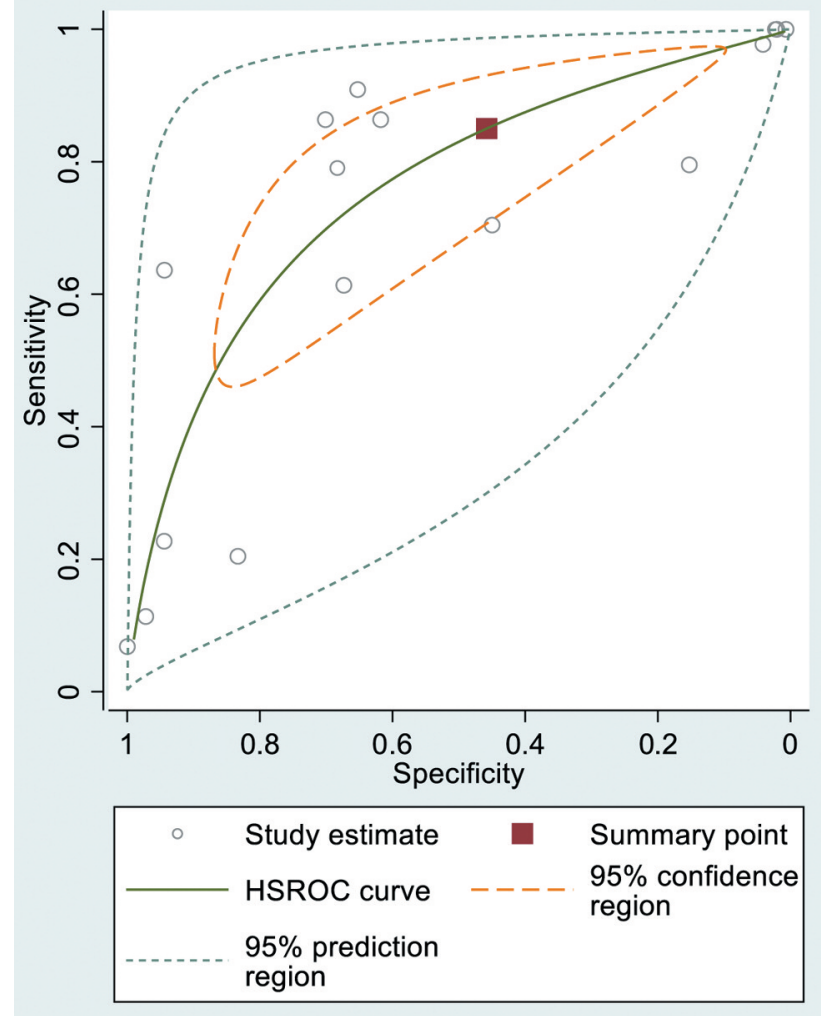

Figure 8. Hierarchical summary receiver operating characteristic (HSROC) curve of the signs and tests.

overall $\mathrm{Sp}$ of the DS in children, $88 \%$ (95\% CI=85-91\%), was slightly higher than that in adult female and male AA patients; $85 \%(95 \% \mathrm{CI}=74-94 \%)$ and $84 \% \quad(95 \% \mathrm{CI}=74-92 \%)$, respectively. Interestingly, the AUC values based on HSROC analysis of the DS in children [0.952 (95\% CI=0.930-0.968)] in female $[0.953(95 \% \mathrm{CI}=0.923-0.969)]$ and male patients [0.956 (95\% CI=0.930-0.969)] were very similar.

AUC values based on HSROC comparison test shows that the diagnostic performance of the clinical signs and tests is slightly better than that of the clinical symptoms only $(p=0.0055)$. However, as measured by the AUC values, the DS formula is superior to both i) the clinical symptoms and ii) signs/clinical tests. Recently, Mujica-Guevara et al. (18) validated the Pediatric Appendicitis Score (PAS) and the Lintula Score $(8,9)$ for AA in children, obtaining AUC values of 0.924 and 0.942 , respectively. These authors evaluated a prospective series of 4-15-year-old children admitted to the pediatric emergency department due to AAP. Both scores were rated in each child, and definitive AA diagnosis was confirmed after clinical follow-up or histopathological examination of the appendectomy specimen (18). Altogether, 100 AAP patients were included, with 47 children having confirmed AA. Based on the ROC 


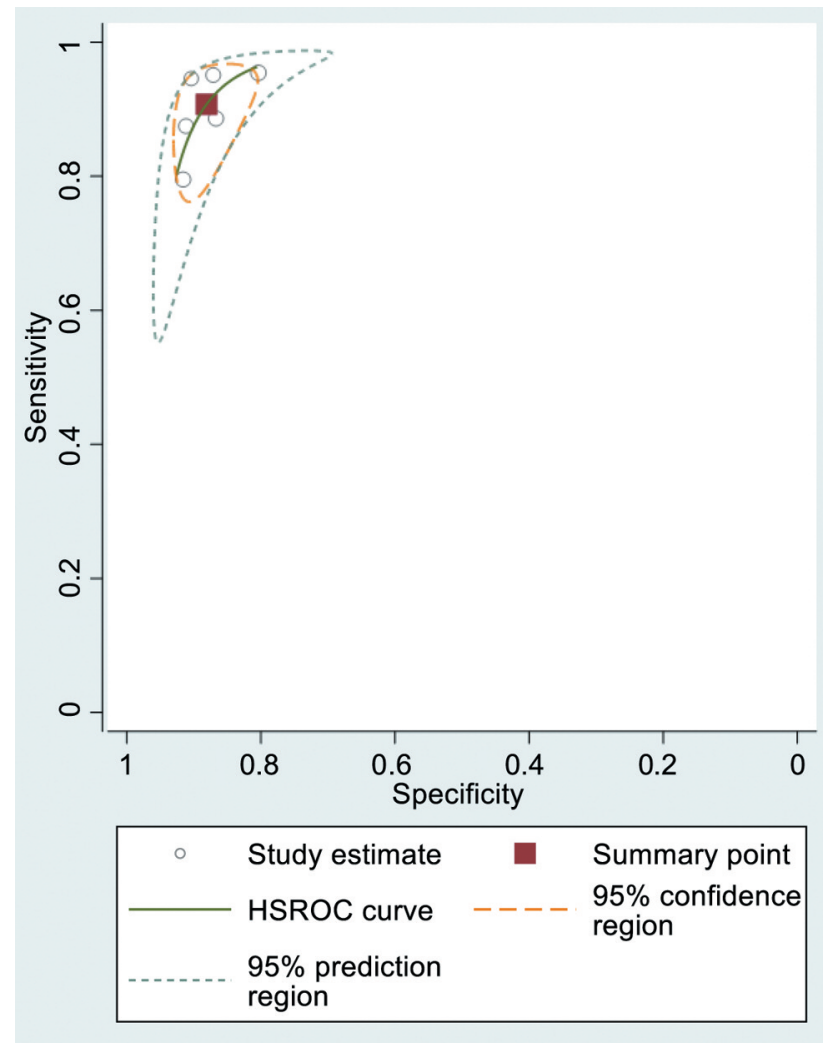

Figure 9. Hierarchical summary receiver operating characteristic (HSROC) curve of the six DS.

curve analysis, the optimal cut-off level for the Lintula Score was 20 and that for the PAS score was 6 . The authors found no significant differences between these two DS, and encouraged clinicians to use the DS in the clinical evaluation of children with AAP (18).

The present study specifically focused on HSROC analysis and AUC value as the most important DS test characteristics. The main objective of the DS is to separate those children who do need immediate attention from those who do not need urgent management. Previous studies have emphasized the value of LC analysis in AA diagnosis, but LC has certain limitations, including i) variation in the definition of LC cutoff, as well as ii) variations in the duration of abdominal pain in the time of LC testing (19). Previously introduced DS systems including PAS (20), Alvarado (3) and APPEND score (21), all require LC analysis. Our DS differed from those in that LC counting is not needed. Although, the LC analysis is not among the independent predictors of AA in the DS formula, the LC may support the AA diagnosis in children, although the Se and Sp of LC analysis are not particularly high as confirmed in the present series; 79\% (95\% CI 64$90 \%$ ) and $68 \%$ (95\% CI 59-77\%), respectively. Furthermore, the diagnostic set-up of AA in children could include early markers of inflammation e.g. blood levels of interleukins (IL6, IL-8 and IL-10) which were shown to increase even 3-fold in perforated AA (22). However, the current enzyme-linked immunosorbent assay (ELISA) rules out its use as a rapid (POC, point-of-care) test in AA in children $(23,24)$.

While considering the limitations of our present study, it is to be admitted that the quite low number of AA children $(n=44)$ among all AAP children $(n=188)$ may affect the final results. Therefore, this should be taken in consideration in planning AAP studies in children in the future.

\section{Conclusion}

Taken together, our novel DS formula, constructed by including the significant independent predictors disclosed by a multivariate analysis, reached very high diagnostic accuracy [Se/Sp balance; AUC $=0.952(95 \% \mathrm{CI}=0.930-0.968)]$ in AA among children. Compared with the diagnostic performance of the clinical findings, signs and tests (ROC comparison test), the DS proved to be far superior to both these conventional diagnostic tools in diagnosis of AA in children.

\section{Conflicts of Interest}

The Authors report no conflicts of interest or financial ties to disclose. The Authors alone are responsible for the content and writing of this article.

\section{Authors' Contributions}

All Authors contributed to the collection and analysis of data, drafting and revising the manuscript, read and approved the final manuscript.

\section{Acknowledgements}

The study was funded by the Päivikki and Sakari Sohlberg Foundation.

\section{References}

1 Bundy DG, Byerley JS, Liles EA, Perrin EM, Katznelson J and Rice HE: Does this child have appendicitis? JAMA 298: 438451, 2007. PMID: 17652298. DOI: 10.1001/jama.298.4.438

2 Ferris M, Quan S, Kaplan BS, Molodecky N, Ball CG, Chernoff GW, Bhala N, Ghosh S, Dixon E, Ng S and Kaplan GG: The global incidence of appendicitis: a systematic review of population-based studies. Ann Surg 266: 237-241, 2017. PMID: 28288060. DOI: $10.1097 /$ SLA.0000000000002188

3 Alvarado A: A practical score for the early diagnosis of acute appendicitis. Ann Emerg Med 15: 557-564, 1986. PMID: 3963537. DOI: 10.1016/s0196-0644(86)80993-3

4 Ohmann C, Franke C and Yang Q: Clinical benefit of a diagnostic score for appendicitis: results of a prospective interventional study. German study group of acute abdominal pain. Arch Surg 134: 993-996, 1999. PMID: 10487595. DOI: 10.1001/archsurg.134.9.993 
5 Sitter H, Hoffmann S, Hassan I and Zielke A: Diagnostic score in appendicitis. Validation of a diagnostic score (Eskelinen score) in patients in whom acute appendicitis is suspected. Langenbecks Arch Surg 389: 213-218, 2004. PMID: 14624293. DOI: $10.1007 / \mathrm{s} 00423-003-0436-9$

6 Lintula H, Pesonen E, Kokki H, Vanamo K and Eskelinen M: A diagnostic score for children with suspected appendicitis. Langenbecks Arch Surg 390: 164-170, 2005. PMID: 15723233. DOI: $10.1007 / \mathrm{s} 00423-005-0545-8$

7 Tzanakis NE, Efstathiou SP, Danulidis K, Rallis GE, Tsioulos DI, Chatzivasiliou, Peros G and Nikiteas NI: A New approach to accurate diagnosis of acute appendicitis. World J Surg 29: 11511156, 2005. PMID: 16088420. DOI: 10.1007/s00268-005-7853-6

8 Lintula H, Kokki H, Kettunen R and Eskelinen M: Appendicitis score for children with suspected appendicitis. A randomized clinical trial. Langenbecks Arch Surg 394: 999-1004. 2009. PMID: 18841382. DOI: 10.1007/s00423-008-0425-0

9 Lintula H, Kokki H, Pulkkinen J, Kettunen R, Gröhn O and Eskelinen M: Diagnostic score in acute appendicitis. Validation of a diagnostic score (Lintula score) for adults with suspected appendicitis. Langenbecks Arch Surg 395: 495-500, 2010. PMID: 20379739. DOI: 10.1007/s00423-010-0627-0

10 Frountzas M, Stergios K, Kopsini D, Schizas D, Kontzoglou K and Toutouzas K: Alvarado or RIPASA score for diagnosis of acute appendicitis? A meta-analysis of randomized trials. Int J Surg 56: 307-314, 2018. PMID: 30017607. DOI: 10.1016/j.ijsu.2018.07.003

11 de Dombal FT: The OMGE acute abdominal pain survey. Progress report 1986. Scand J Gastroenterol 23(Suppl 144): 3542, 1988. PMID: 3043646.

12 Eskelinen $\mathrm{M}$ and Lipponen P: Usefulness of history-taking in non-specific abdominal pain: A prospective study of 1333 patients with acute abdominal pain in Finland. In Vivo 26: 335 339, 2012. PMID: 22351680.

13 Meklin J, Eskelinen M, Syrjänen K and Eskelinen M: Leucocyte count does not improve the diagnostic performance of a diagnostic score (DS) in distinguishing acute appendicitis (AA) from non-specific abdominal pain (NSAP). In Vivo 34: $3327-$ 3339, 2020. PMID: 33144440. DOI: 10.21873/invivo.12171

14 Meklin J, Eskelinen M, Syrjänen K and Eskelinen M: Genderspecific performance of a diagnostic score (DS) in acute appendicitis (AA). In Vivo 34: 3687-3703, 2020. PMID: 33144486. DOI: 10.21873 /invivo. 12217

15 Gharib H: Review: symptoms, signs, and lab tests have moderate accuracy for detecting appendicitis in children. Evid Based Med 13: 23, 2008. PMID: 18234931. DOI: 10.1136/ebm.13.1.23
16 Wyer PC: Review: symptoms, signs, and lab tests have moderate accuracy for detecting appendicitis in children. Arch Dis Child Educ Pract Ed 94: 95, 2009. PMID: 19460902.

17 Sencan A, Aksoy N, Yıldız M, Okur Ö, Demircan Y and Karaca I: The evaluation of the validity of Alvarado, Eskelinen, Lintula and Ohmann scoring systems in diagnosing acute appendicitis in children. Pediatr Surg Int 30: 317-321, 2014. PMID: 24448910. DOI: 10.1007/s00383-014-3467-0

18 Mujica-Guevara JA, Pierdant-Pérez M, Gordillo-Moscoso AA, Martínez-Martínez UM and Ramírez-Meléndez R: Comparison of PAS and Lintula score in diagnosis suspect of appendicitis in children. Acta Pediatr Mex 39: 209-215, 2018.

19 Stefanutti G, Ghirardo V and Gamba P: Inflammatory markers for acute appendicitis in children: are they helpful? J Pediatr Surg 42: 773-776, 2007. PMID: 17502181. DOI: 10.1016/j.jpedsurg. 2006.12.028

20 Samuel M: Pediatric appendicitis score. J Pediatr Surg 37: 877881, 2002. PMID: 12037754. DOI: 10.1053/jpsu.2002.32893

21 Andersson M and Andersson RE: The appendicitis inflammatory response score: a tool for the diagnosis of acute appendicitis that outperforms the Alvarado score. World J Surg 32: 1843-1849, 2008. PMID: 18553045. DOI: 10.1007/s00268-008-9649-y

22 Anielski R, Kúńnierz-Cabala B and Szafraniec K: An evaluation of the utility of additional tests in the preoperative diagnostics of acute appendicitis. Langenbecks Arch Surg 395: 1061-1068, 2010. PMID: 19924436. DOI: 10.1007/s00423-009-0565-x

23 Aspinen S, Kinnunen M, Harju J, Juvonen P, Selander T, Holopainen A, Kokki H, Pulkki K and Eskelinen M: Inflammatory response to surgical trauma in patients with minilaparotomy cholecystectomy versus laparoscopic cholecystectomy: a randomised multicentre study. Scand J Gastroenterol 51: 739-744, 2016. PMID: 26758677. DOI: 10.3109/00365521.2015.1129436

24 Purdy M, Kokki M, Anttila M, Aspinen S, Juvonen P, Korhonen R, Selander T, Kokki H and Eskelinen M: Does the rectus sheath block analgesia reduce the inflammatory response biomarkers' IL-1ra, IL-6, IL-8, IL-10 and IL-1 $\beta$ concentrations following surgery? A randomized clinical trial of patients with cancer and benign disease. Anticancer Res 36: 3005-3011, 2016. PMID: 27272818 .

Received November 25, 2020

Revised December 3, 2020

Accepted December 8, 2020 